\title{
Exploring Muscular Contribution during Stepping of Biomimetic Feline Hindlimbs
}

\author{
Andre Rosendo, Shogo Nakatsu, Kenichi Narioka and Koh Hosoda
}

\begin{abstract}
Although robotic locomotion have greatly advanced over the past years, the abyss that separates such locomotion from even the simplest animal locomotions prompt us to approach robotic locomotion taking cues from animals. The animal musculoskeletal structure, often ignored by roboticists due to its high redundancy and complexity, might hold the secret for self-stable locomotion observed in bipeds and quadrupeds. Aiming to better understand how muscles contribute to selfstable locomotion we take the feline structure as a model on a biomimetic approach. Using 6 air muscles per hindlimb to mimic real muscles, this robot walks stably on a treadmill while supported by a slider, simulating forelimbs. We individually evaluate muscle contribution to walking stability, performing a comparison between mono and biarticular synergistic muscles at the ankle and concluding that a higher compliance on the biarticular muscle improved walking stability. A better understanding of such complex phenomena may help on the development of better legged robots in the future, truly taking advantage of concepts developed by nature over the years.
\end{abstract}

\section{INTRODUCTION}

Over the years, robotic locomotion has improved itself with walking, running and even swimming robots ([1], [2], to name a few). Roboticists have been working in powerful control methods which predict the body attitude and generates a leg trajectory, creating a stable gait. Although mathematically sound, these human-made methods produce fairly unnatural gaits, being vastly outperformed in every aspect by strides of simple living forms, such as cockroaches or mice.

Observing natural phenomena is the oldest and most famous source of scientific discoveries. Biologists have been observing animal locomotion for years, trying to correlate electrical signals between brain and muscles with the angular motion output on joints. Engberg and Lundberg [3], Goslow [4] [5], English [6] and Herzog [7] are examples of researchers which allowed a higher understanding of feline and canine locomotion. Studying and understanding complex structures, such as the musculoskeletal system of fore and hindlimbs, might help explaining the huge performance gap between current robots and animals.

Albeit the tiny brain size of a cockroach, mouse or even a cat, their highly adaptive behavior is far superior than current robotic research. Experiments with decerebrate cats [8] contribute to the conclusion that the aforementioned complex leg might prescind from higher level control, relying on a more fundamental rule to walk.

This work was partially supported by KAKENHI Kiban(S) 23220004

The authors are with Multimedia Department, Faculty of Information Science and Technology, Osaka University, 1-5 Yamadaoka, Suita, Osaka, Japan. andre.rosendodist.osaka-u.ac.jp
Over the years many quadruped $\operatorname{robots}($ e.g., [9] [10]) have been developed. Aiming to develop better robots through a different approach, a few researchers have been trying to tackle animal locomotion by biomimicking. Works such as [11] allows the study of a cheetah by simply reproducing movements with electric motors, while works from [12] and [13] adopted monoarticular pneumatic muscles as actuation means. In [14] a cheetah-like robot is designed, adopting exclusively biarticular muscles for actuation, while in [15] a quadruped robot with mono and biarticular muscles uses a simplified form of locomotion to crawl.

To date, the most significant works to replicate quadrupedal locomotion considering a musculoskeletal perspective were [16] and [17]. Both groundbreaking works consider two feline hindlimbs attached to hips and walking on a simulated environment, drawing conclusions regarding muscle contribution to walking stability and proposing a ground unloading rule, responsible for generating an alternating stepping on a quadrupedal walking.

This work focuses on recreating the same feline stepping experiment suggested by Ekeberg [16] in a real world environment. Although simulations can help scientists grasp the outcome of a proposed experiment, many variables associated to the real world are often relaxed, such as noise and slippage. Upon adopting a robot with 6 muscles on each hindlimb, here called Pneupard [18], we could verify that the ground unloading rule proposed by Ekeberg holds true against real world environment. Further, contributions to walking stability of synergistic pairs at the ankle are briefly analyzed.

Differently from other robotic works, this work does not intend to create a self-contained walking robot. Pneupard aims to understand how muscles contribute to self-stability, knowledge which allows a more through understanding of how animals achieve a remarkable performance during locomotion. In the future, such knowledge may help on the development of robots capable of adapting to urban environments, full of obstacles and disturbances.

\section{MATERIALS AND METHODS}

Similarly to works from other cheetah-inspired robots [11] [14], the design of Pneupard is based on extracted data from domestic cats (Felis catus) and cheetahs (Acinonyx jubatus). Forelimbs, hindlimbs and inter-girdle measurements were considered from a comparative study between cats with different proportions [19], while muscular arrangement and choice (due to the higher number of muscles, only the most 


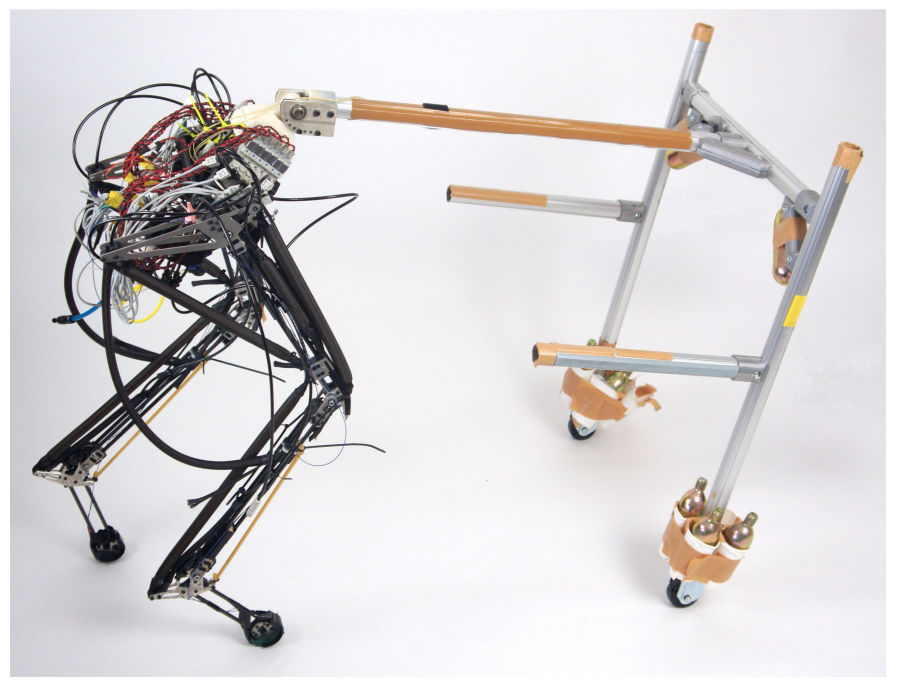

Fig. 1. Feline hindlimbs attached to sliding strut. This experimental setting allows walking experiments on a treadmill.

representative ones were chosen) were based on published data with cheetah cadavers [20] [21].

Each hindlimb possess 6 active muscles and 2 passive muscles. A main difference between Pneupard and other robots created so far is the focus on exploring the highly redundant muscular structure observed in animals $(e . g$, humans have soleus, plantaris and gastrocnemius performing the same ankle extension function, but used separately). A picture of the experimental assembly for Pneupard can be found is shown in Fig. 1.

Thus, the 6 existing muscles are separated in 3 synergistic pairs on ankle, knee and hip, as follows:

- Hip joint: Hip angular changes are controlled by 1 passive and 2 active muscles. While flexor movements are controlled by an elastic rubber which simulates a hip flexor, such as iliopsoas (IL), extensor movements of the hip are either actuated by a monoarticular muscle (inserting at the femur, such as biceps femoris (BF)) or a biarticular muscle (inserting at the tibia, slightly below the knee joint, similarly to semimenbranosus (SM)).

- Knee joint: The knee joint possess 2 synergistic muscles. A monoarticular muscle attached between femur and tibia, similarly to vastus lateralis (VL), allows solely flexion of the knee, while a second muscle, originated from the hip, allows not only knee extension but also hip flexion, similarly to rectus femoris (RF).

- Ankle joint: Ankle extension movements are created by one monoarticular and one biarticular muscle. The monoarticular, similarly to soleus (SO), originates from the tibia, pulling the heel up, while the biarticular performs the same movement, originating from the femur, above the knee joint, similarly to gastrocnemius (GA). Flexing movements are simulated by an elastic playing the role of tibialis anterior (TA).

The hindlimbs are made of carbon fiber, ABS plastic and magnesium alloy plates, contributing for a lightweight

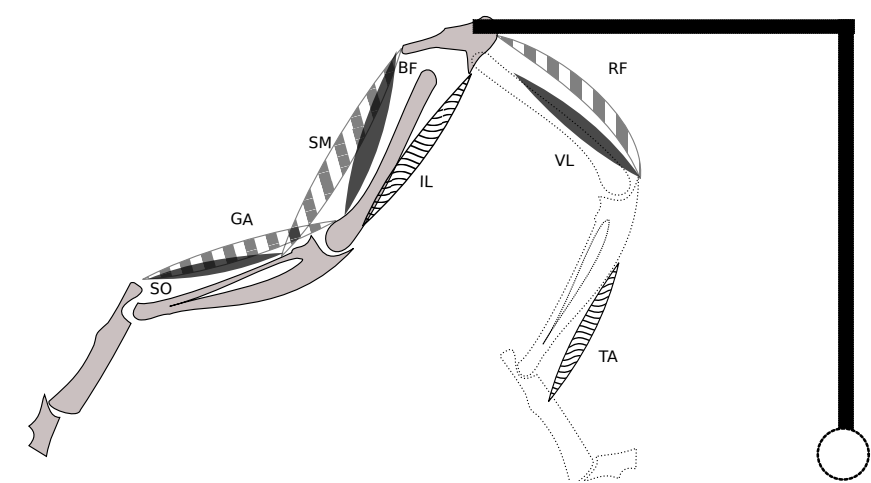

Fig. 2. Pictogram of Pneupard's muscles. Biarticulars are depicted with thick stripes, monoarticulars in solid pattern and passive muscles with thin stripes.

TABLE I

PNEUPARD'S KEY CHARACTERISTICS

\begin{tabular}{c|c}
\hline Property & Value \\
\hline Hindlimb length & $810 \mathrm{~mm}$ \\
\hline Body width & $300 \mathrm{~mm}$ \\
\hline No of degrees of freedom & 6 \\
\hline No of active muscles & 12 \\
\hline Hindlimb weight & 600 grams \\
\hline Total weight (without strut) & $2.5 \mathrm{~kg}$ \\
\hline
\end{tabular}

system. The overall specs of the robot are depicted on Table I, while a pictogram showing the position of the muscles can be found in Fig. 2.

\section{A. Pneumatic air muscles}

The aforementioned muscles are used as a replacement to biological muscles. Although pneumatic artificial muscles are not real muscles, their behavior is hitherto the closest available to biological muscles, being used by many biomimetic roboticists [1] [22]. Experimental comparisons between pneumatic and biological muscles have shown that force-length properties are similar to biological data [23], being a fair representation if used at low contraction speeds (force-velocity behavior differs from real muscles).

We adopted hand-made air muscles, with an inner rubber tube diameter of $8 \mathrm{~mm}$, thickness of $1 \mathrm{~mm}$ and a nylon braided sheath of $9 \mathrm{~mm}$ diameter surrounding the muscles. Supplying air to the muscle generates a contraction, not only shortening the muscle but also stiffening it (very important to provide different compliance levels during locomotion). Exhausting the air restores the muscle to its natural position, also making it more compliant.

\section{B. Electro-pneumatic interface}

Air muscles require a stable supply of medium-pressure $(0.7 \mathrm{MPa})$ air for a successful walking to take place. Although in the future remote applications considering an onboard compressor or a high-pressure tank are being considered, at the current state a tether supplies the system with 
air and energy (12 VDC). Pneupard's main objective is to provide insights on animal locomotion, being a treadmill experiment sufficient to fulfill this target.

Aiming to build a lightweight body with all pneumatic valves on-board, pilot operated on-ff valves (VQZ1321, SMC Corporation) were chosen over proportional valves. Proportional valves allow a more precise pressure control inside muscles, but usually the price to be paid is low flow rate with cumbersome weight. Using a control method developed in previous experiments [18], controlling pressure with an onoff valve is possible.

Each muscle is connected to a pressure sensor, allowing data acquisition from individual muscles during walking. Each hindlimb has a force sensor at the tip of each leg, allowing a feedback response from the floor. The gait generated by the muscular activation was stable enough, not requiring gyroscopes or accelerometers for walking.

\section{Control and Activation Pattern}

The hindlimbs are controlled through a microcontroller (ARM $76 \mathrm{MHz}$ ), attached to its body and connected to a computer, transmitting data (muscle pressure and force sensor output) at a frequency of $40 \mathrm{~Hz}$.

Using EMG signals from real cats [3], similarly to the method adopted in Ekeberg's simulation [16], we built a finite state control with 4 distinct phases: Lift off (LO), Swing (SW), Touch down (TD) and Stance (ST). Each phase has a target pressure for each muscle, and there is no coupling between right and left hindlimbs. The initial phase is Touch down, where the microcontroller samples for activity on the force sensor at the tip of the hindlimb. When stimulated, the state is switched to Stance, which lasts until the ground reaction force acting on the sensor decreases to a value below a specific threshold, activating then the Lift off phase. The end of the Lift off phase happens when the contact between the sensor and the floor no longer exist, starting the Swing phase.

As seen in Fig. 3, during swing phase every muscle relaxes to allow swing (soleus partially relaxes to avoid overflexing the ankle joint), and during stance phase every muscle is recruited (some constantly recruited, while others progressively, to allow a smoother movement). In progressively recruited muscles ripples can be seen, as an effect of the adopted on-off valve control method. Differently from Ekeberg, Pneupard's swing phase is based on a timer interrupt, not requiring a second sensor on the hip. After the specified time, the hindlimb enters Touch down phase again, waiting for floor contact.

\section{Experimental Method}

Experiments were conducted with a treadmill running at constant speed $\left(0.8 \mathrm{~km} \mathrm{~h}^{-1}\right)$, which was defined after trials with the robot, being this chosen speed the robot's baseline speed. Initially, Ekeberg's activation pattern [16] and biological EMG signals [3] were compared, creating an activation pattern inspired on these levels. After this initial process, fine tuning of the force sensor and muscle pressure

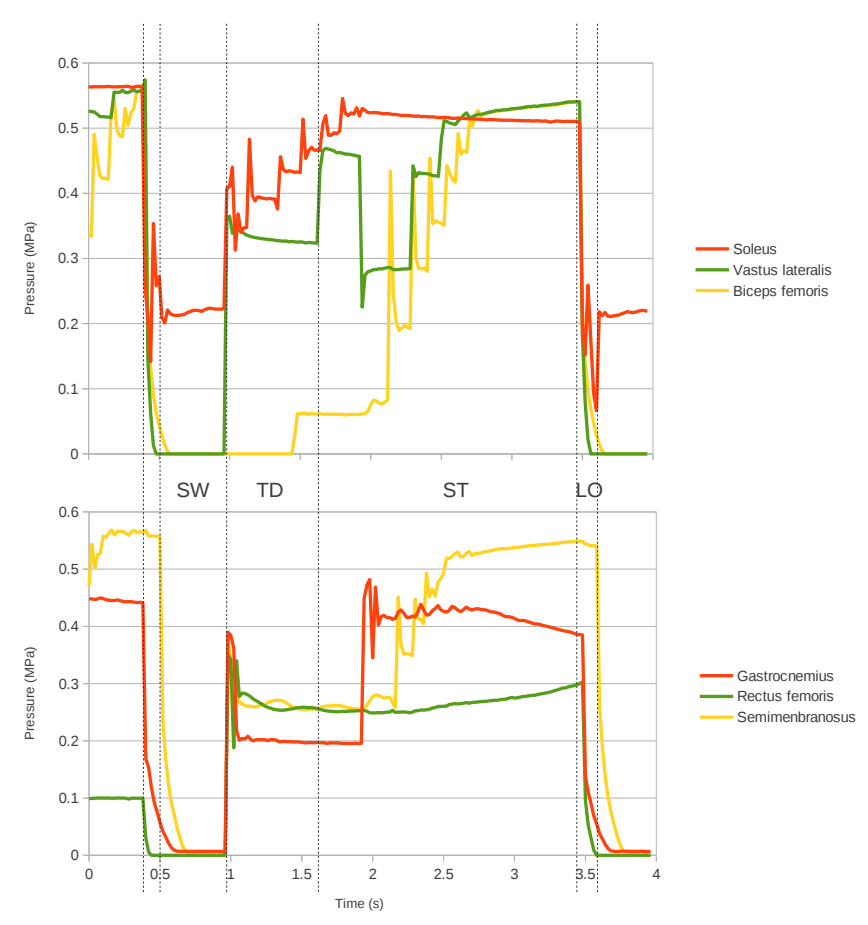

Fig. 3. Activation pattern of hindlimbs, considering real output pressures along the 4 different gait phases. On top we have monoarticular muscles, while on the bottom their biarticular pairs. The gait duration of 4 seconds was set for easier explanation, being faster than this.

took place to reach the most stable walking, thus called baseline.

The experiments aimed to understand individual contributions from ankle muscles on the gait stability during cat stepping, with systematic changes on muscular pressures to find relationships between muscle pressure and gait stability. Initial experiments considered the biggest ankle extensor (gastrocnemius), while additional experiments analyzed this same muscle when compared to the smaller, monoarticulated soleus muscle. As a stability measuring criteria, we registered the influence of these disturbances on the phase difference between right and left hindlimbs. Similarly to Ekeberg, the phase difference is defined by:

$$
\Phi_{H(\text { actual })}=\frac{T_{H(\text { actual })}-T_{O(\text { previous })}}{T_{O(\text { next })}-T_{O(\text { previous })}}
$$

As a success criteria a minimum of 20 steps was established, where walking experiments with less are considered a failure, similarly to the methodology adopted previously [24].

\section{RESULTS}

Initial data acquired from the baseline condition shows the robot walking stably on the treadmill. In Fig. 4 an example of the alternating gait, with force being transfered between right and left hindlimbs is shown. Asymmetries between sensors are responsible for slightly different values among right and left hindlimbs.

Applying the stability criteria between right and left hindlimbs, we can see that slippage plays an important 


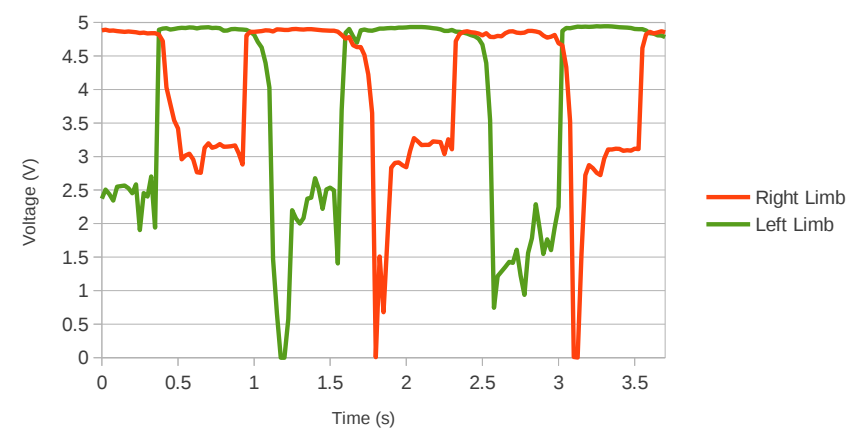

Fig. 4. Force sensor values of robot while walking on the treadmill. These values are used to decide whether the hindlimb is in touching the treadmill (stance or lift off) or not (swing or touch down).

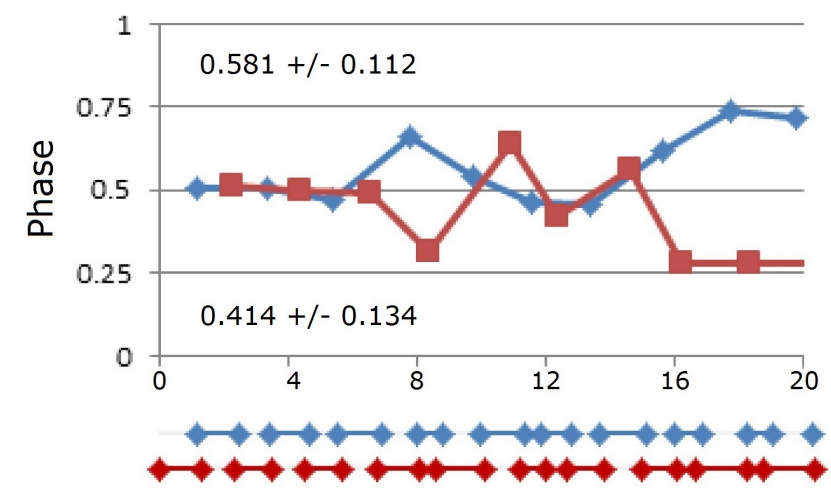

Fig. 5. Walking experiment on a treadmill with baseline settings. Red and blue lines represent right and left hindlimbs, respectively. Mean values and standard deviations are shown for each hindlimb. Natural disturbances occur around 6 and 14 seconds

role on real world robotics: While low friction between hindlimbs and treadmill produce slippage, terminating stance prematurely, high friction occasionally terminates the swing phase early, with the leg touching the floor before the intended time. In Fig. 5 we can see the influence of such natural disturbances on the gait, setting it apart from the center of the graph $(0.5)$.

In order to understand the influence of gastrocnemius on the walking stability, we fixed the baseline parameters and systematically changed the values for gastrocnemius muscle pressure during stance phase. The results of this experiment are shown in Fig. 6. The system presented 2 different failure modes: High pressure failure after walking a few steps and low pressure failure for being incapable of standing up, failing to start the walking experiment.

This second failure mode prompted us to the possibility that more compliant gastrocnemius could generate more adaptive walking if we combined this pressure decrease with a pressure increase on its synergistic pair (soleus). Thus, we conducted new experiments, adjusting the pressures in soleus and in gastrocnemius accordingly.

The results shown in Fig. 7 consider a trade-off between soleus and gastrocnemius during walking. For every 0.06 $\mathrm{MPa}$ increment given to soleus, the same values were re-

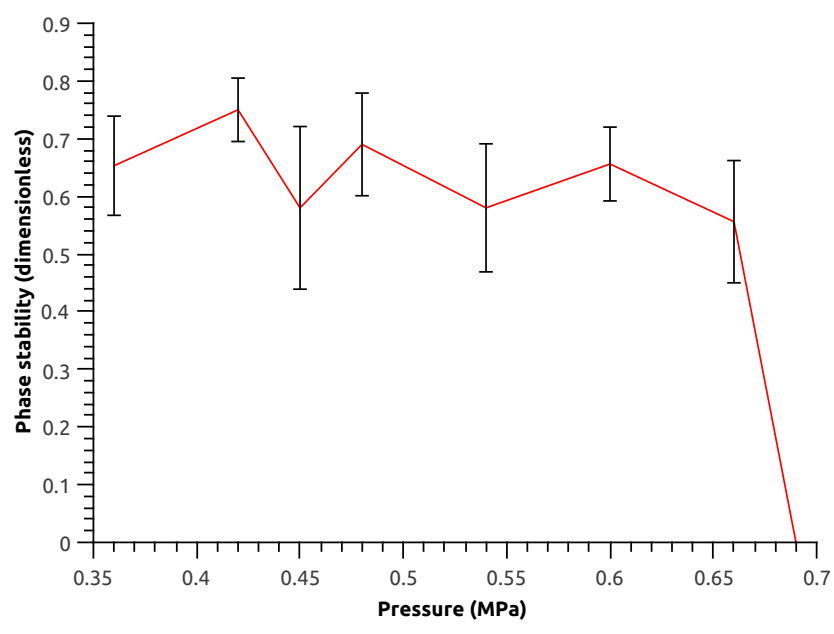

Fig. 6. Mean and standard deviation values for systematic pressure changes on gastrocnemius muscle during walking. Best results should remain close to the 0.5 value range. Only the left hindlimb is depicted in this experiment.
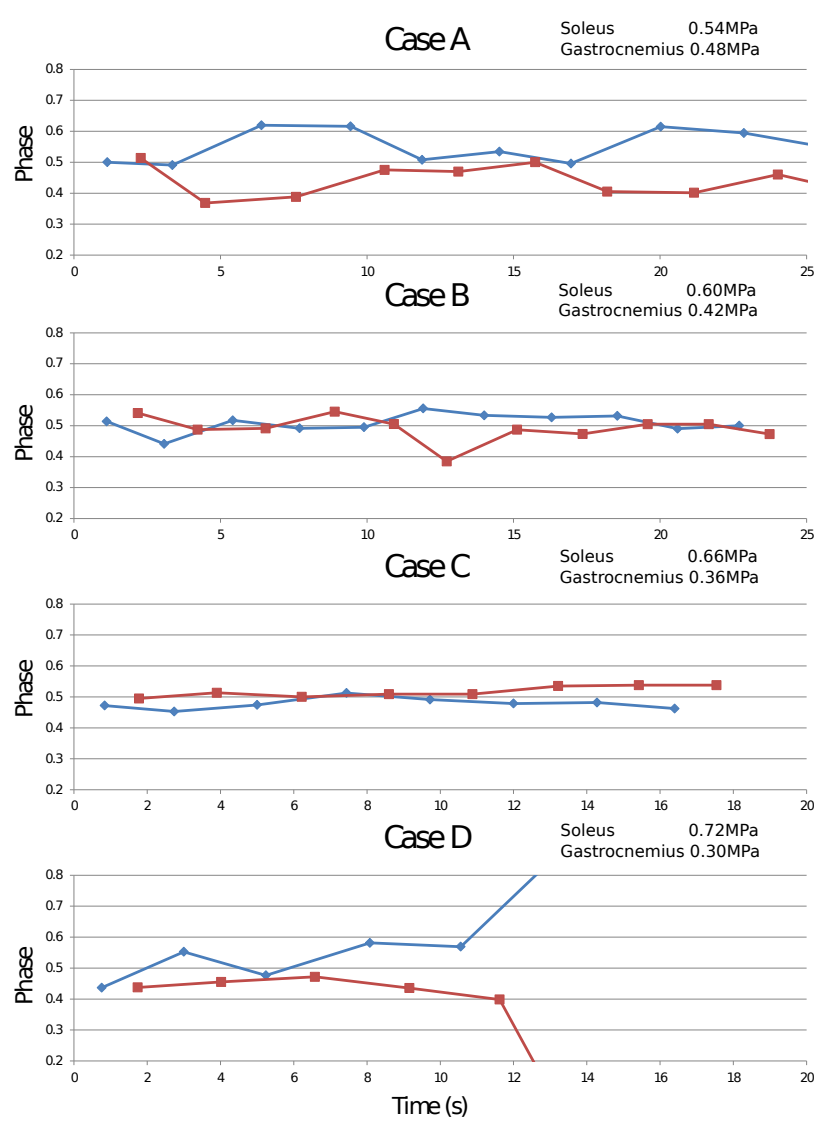

Fig. 7. Evaluation of synergy between gastrocnemius and soleus. The 4 different cases have different pressure settings for each muscle.

moved from gastrocnemius. Considering that both muscles are attached at the same place at the heel (same varying moment arm), the final ankle torque should remain unchanged.

\section{DISCUSSION}

In Fig 4 right and left hindlimbs alternate the body load between each other in a stable walking, without flight phases. 
In Fig. 5 the gait starts with both legs very close to the 0.5 mark, drifting away and returning after some random disturbance on the treadmill. Although the 0.5 mark stands for the optimum gait (perfectly symmetric), it is important to note that drifting away from the 0.5 mark does not necessarily mean that the gait is unstable. This is particularly true when comparing simulations with real world experiments, where the former has all variables perfectly controlled and the latter suffers from countless noise sources.

\section{A. Gastrocnemius Role on Walking}

Experiments with gastrocnemius have shown that stability values for gastrocnemius do not show any specific trend with increasing or decreasing pressure. From Fig. 6 we can infer that the lack of gastrocnemius affected the robot negatively, not being capable of standing or walking. Similarly, too much pressure on gastrocnemius renders it over stiff, dramatically degrading the touch down phase of the opposite limb (over contracted muscles increase stance height).

We test the hypothesis of soleus complementing the ankle torque with a softer gastrocnemius in Fig. 7. Interestingly, the fact that a stronger soleus was supporting gastrocnemius did not change the outcome of the walking, with the robot still failing to walk at $0.3 \mathrm{MPa}$ (similarly to the previous experiment).

The muscle gastrocnemius is located in a very strategic position: As an ankle extensor it receives the highest impacts from the floor, while as a knee flexor it synchronizes knee and ankle movements. Thus, similarly to a compliant four bar linkage, gastrocnemius deforms when solicited and coordinates movements when not touching the floor. Since some compliance is necessary, lower pressure values resulted in better performance until failure. Being a fast twitching muscle, on the real animal compliance can be rapidly adjusted to match the appropriate gait/speed.

\section{B. Synergistic contribution to stability}

While performing experiments with synergistic pairs (Fig. 7), we could notice a tendency as soleus pressure increased and gastrocnemius pressure decreased. Observing cases A, B and $C$ we can infer that stability improved with the changes, getting closer and closer to the 0.5 line. If simply decreasing gastrocnemius would be the source of such improvement, then this same improvement should have occurred in Fig. 6. (lowering gastrocnemius, closing on the 0.5 mark)

The increase in soleus pressure indicates that the ankle total torque, which should decrease by the lack of gastrocnemius contribution, is being compensated by soleus, but the same is not true when we consider knee torque. Lowering gastrocnemius means that the knee flexing contribution decreases, making it easier for vastus lateralis and rectus femoris to extend the knee. Moreover, a more compliant gastrocnemius allows a "spring-like" behavior of the four bar linkage, while a "slack" gastrocnemius does not offer the four bar linkage feature discussed above.

Thus, while we can infer that in cases $\mathrm{A}, \mathrm{B}$ and $\mathrm{C}$ the compliance present in gastrocnemius is ideal to coordinate angular movements between ankle and knee joints, gastrocnemius values below $0.35 \mathrm{MPa}$ (Case D) rendered the muscle so soft that no coordination between knee and ankle existed.

\section{CONCLUSIONS AND FUTURE WORKS}

The morphological complexity of animals is a conundrum which roboticists are still scratching the surface, and this work delves into the problem aiming to provide more clarity on the subject.

In this work we developed a walking robot with 2 hindlimbs attached to a sliding strut. Based on biological concepts, this robot main purpose is to help close the gap between animal and robotic locomotion. We performed experiments in a treadmill (Fig. 8) and tested the influence of the muscle gastrocnemius on the walking stability. Moreover, we tested the influence of the synergistic pair gastrocnemius/soleus on the walking stability, reaching the conclusion that a more compliant gastrocnemius allows greater stability during walking.

Future works will focus on reproducing different types of locomotion (bounding, trotting) with a fully assembled quadruped robot, currently under final phase of development. As a long-term objective, we hope that this platform allows us to better understand cats, replicating these concepts on better robots in the future.

\section{REFERENCES}

[1] K. Hosoda, Y. Sakaguchi, H. Takayama and T. Takuma, "Pneumaticdriven jumping robot with anthropomorphic muscular skeleton structure", Auton. Robot., Vol. 28, 2010, pp. 307-316.

[2] A. Rosendo, S. Nakatsu, K. Narioka and K. Hosoda, "Toward a stable biomimetic walking: Exploring muscle roles on a feline robot", in Proc. Sixth Intl. Symp. Adaptive Motion on Animals and Machines, Darmstadt, Germany, 2013.

[3] I. Engberg and A. Lundberg, "An electromyographic analysis of muscular activity in the hindlimb of the cat during unrestrained locomotion", Acta Physiol. Scand., Vol. 75, 1969, pp. 614-630.

[4] G.E. Goslow Jr., R.M. Reinking and D.G. Stuart, "The cat step cycle: hind limb joint angles and muscle lengths during unrestrained locomotion", J. Morphol., Vol. 141, 1973, pp. 1-42.

[5] S. Rasmussen, A.K. Chan and G.E. Goslow Jr., "The cat step cycle: electromyographic patterns for hindlimb muscles during posture and unrestrained locomotion", J. Morphol., Vol. 155, 1978, pp. 253-269.

[6] A. English, "An electromyographic analysis of forelimb muscles during overground stepping in the cat", J. Exp. Biol., Vol. 76, 1978, pp. 105-122.

[7] B.I. Prilutsky, W. Herzog and T. Leonard, "Transfer of mechanical energy between ankle and knee joints by gastrocnemius and plantaris muscles during cat locomotion", J. Biomech., Vol. 29, 1996, pp. 391403.

[8] G.W. Hiebert, K.G. Pearson, "Contribution of sensory feedback to the generation of extensor activity during walking in the decerebrate cat", J. Neurophysiol., Vol. 81, 1999, pp. 758-770.

[9] N.G. Tsagarakis, B. Vanderborght, Y. Yousheng, and D.G. Caldwell, HyQ - Hydraulically actuated quadruped robot: Hopping leg prototype, in Proc. Int. Conf. Biomed. Robot. and Biomechatronics, 2008, pp. 593-599.

[10] Y. Fukuoka, H. Kimura and A.H. Cohen, ”Adaptive dynamic walking of a quadruped robot on irregular terrain based on biological concepts", J. Robot. Res., Vol. 22, 2003 , pp. 187-202.

[11] A. Sproewitz, A. J.Ijspeert et al., "Oncilla robot : a light-weight bioinspired quadruped robot for fast locomotion in rough terrain", in Proc. Fifth Intl. Symp. Adaptive Motion on Animals and Machines, Osaka, Japan, 2011. 

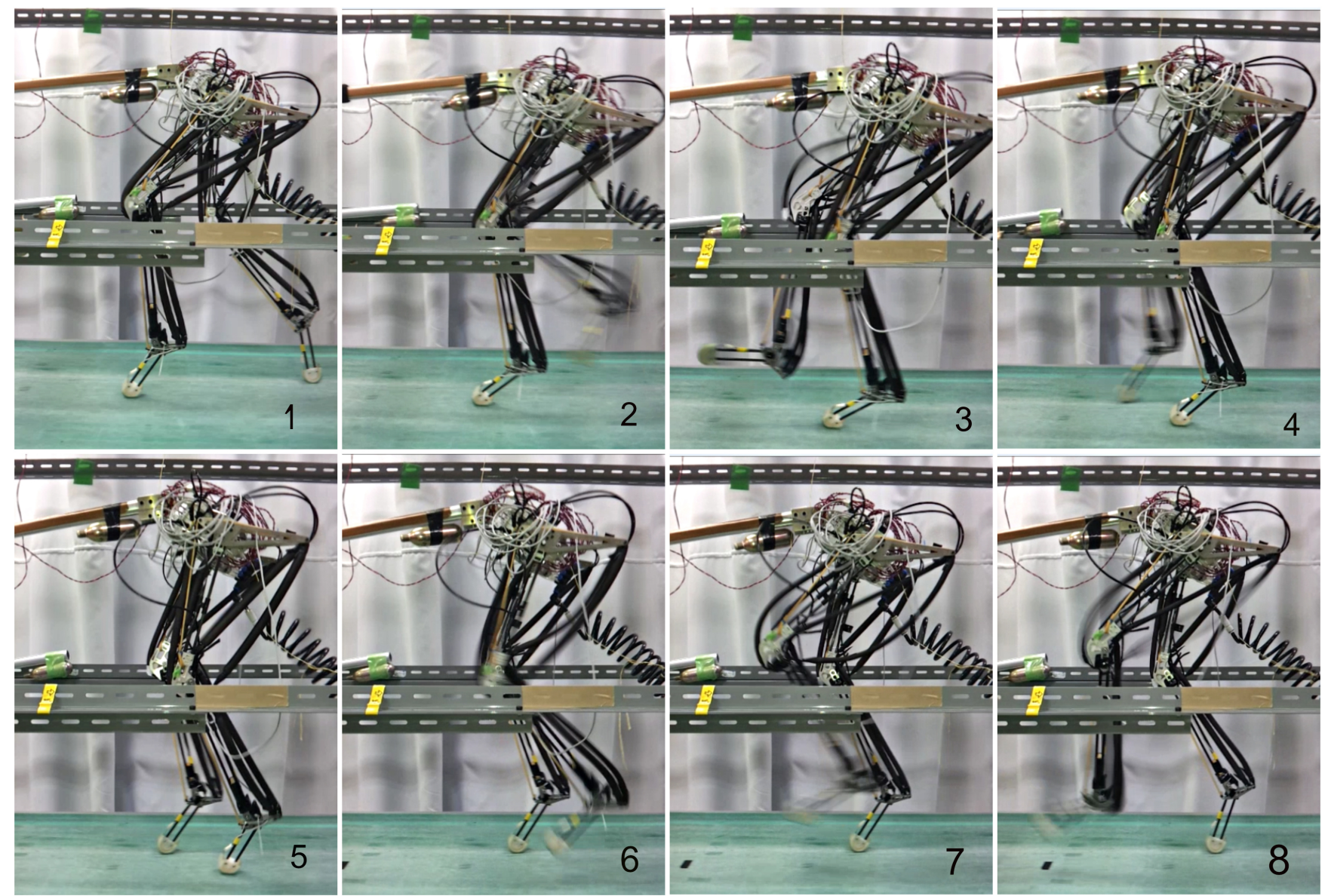

Fig. 8. Snapshots of Pneupard walking on the treadmill. In the first 4 snapshots we have the right leg swinging while the left stays in stance phase. The following 4 snapshots depict the opposite situation.

[12] K. Tsujita, T. Kobayashi, T. Inoura and T. Masuda, "Gait transition by tuning muscle tones using pneumatic actuators in quadruped locomotion", in Proc. Intl. Conf. on Intelligent Robots and Systems, 2008, pp. 2453-2458.

[13] K. Aschenbeck, N. Kern, R. Bachmann and R. Quinn, ”Design of a quadruped robot driven by air muscles" in Proc. Intl. Conf. Biomed. Robot. and Biomechatronics, 2006, pp. 875-880.

[14] M. Lewisy, M. Buntingy, B. Salemi and H. Hoffmann, "Toward ultra high speed locomotors: design and test of a cheetah robot hind limb", in Proc. Intl. Conf. on Robotics and Automation, 2011, pp. 1990-1996.

[15] Y. Yamada, S. Nishikawa, Y. Kuniyoshi et al., Neural-body coupling for emergent locomotion: a musculoskeletal quadruped robot with spinobulbar model, in Proc. Int. Conf. on Intelligent Robots and Systems, 2011, pp. 1499-1506.

[16] O. Ekeberg and K. Pearson, "Computer simulation of stepping in the hind legs of the cat: an examination of mechanisms regulating the stance-to-swing transition", J. Neurophysiol., Vol. 94, 2005 , pp. 42564268.

[17] S. Yakovenko, V. Gritsenko and A. Prochazka, "Contribution of stretch reflexes to locomotor control: a modeling study", Biol. Cybern., Vol.
90, 2004, pp. 146-155.

[18] A. Rosendo, K. Narioka, and K. Hosoda, "Muscle roles on directional change during hopping of a biomimetic feline hindlimb", in Proc. Int. Conf. on Robotics and Biomimetics, 2012.

[19] L. Day and B. Jayne, Interspecific scaling of the morphology and posture of the limbs during the locomotion of cats (Felidae), J. Exp. Biol., vol. 210, 2007, pp. 642-657.

[20] P.E. Hudson, A.M. Wilson et al., Functional anatomy of the cheetah (Acinonyx Jubatus) hindlimb, J. Anat., vol. 218, 2011, pp. 363-374.

[21] P.E. Hudson, A.M. Wilson et al., Functional anatomy of the cheetah (Acinonyx Jubatus) forelimb, J. Anat., vol. 218, 2011, pp. 375-385.

[22] I. Boblan, "A human-like robot hand and arm with fluidic muscles: biologically inspired construction and functionality", Embodied Artficial Intelligence, Springer, Dagstuhl Castle, 2004.

[23] Klute G, Czerniecki J, Hannaford B. "Artificial muscles: Actuators for biorobotic systems", Int. J. Robotics Res., vol. 21, 2002, pp. 295-309.

[24] A. Rosendo, S. Nakatsu, K. Narioka and K. Hosoda, "Pneupard: A biomimetic musculoskeletal approach for a feline-inspired quadruped robot", in Proc. Int. Conf. on Intelligent Robots and Systems, 2013, in press. 